\title{
FPSO RISK ASSESSMENT AND ACCEPTANCE CRITERIA WITH APPLICATION TO FPSO MOORING SYSTEMS
}

\author{
Michael H. Faber \\ ETH Zurich \\ Institute of Structural Engineering IBK \\ ETH-Hönggerberg, $\mathrm{CH}-8093$ Zürich \\ Switzerland \\ faber@ibk.baug.ethz.ch
}

\author{
Roberto Montes-Iturrizaga \\ Instituto Mexicano del Petróleo, \\ Programa de Aguas Profundas, \\ México DF, México \\ eheredia@imp.mx
}

\author{
Daniel Straub \\ Matrisk GmbH \\ Zürich, Switzerland \\ ds@matrisk.com
}

\author{
Ernesto Heredia-Zavoni \\ Instituto Mexicano del Petróleo, \\ Programa de Aguas Profundas, \\ México DF, México \\ rmontes@imp.mx
}

Keywords: Risk assessment, Bayesian Probabilistic Nets, PFSO risk scenarios, risk acceptance criteria, the LQI principle, mooring systems, target reliability.

\footnotetext{
ABSTRACT

Risk based design code safety format calibration for FPSO installations is addressed based on the results of a recent industrial project performed for IMP by the authors. Generic risk models for general engineering systems are introduced taking basis in recent work by the Joint Committee on Structural Safety (JCSS). Thereafter, the scenarios considered for the risk based calibration of a design code safety format for the design of FPSO facilities in the Gulf of Mexico are outlined in some detail. Furthermore, it is shown how these scenarios may be represented in a generic risk assessment model greatly enhanced by the utilization of hierarchical risk modeling procedures such as Bayesian Probabilistic Nets (BPN's). A short outline of the Life Quality Index (LQI) principle is then introduced as a practically applicable means to determine how much should be invested into life saving activities. Finally, an example is given where the introduced concepts are used for the purpose of determining the target reliability index for individual mooring lines taking into account the direct and
}

indirect consequences of failure of the mooring system for a considered generic FPSO installation.

\section{INTRODUCTION}

As the Mexican oil industry plans to move towards production in deep water, it is necessary to develop criteria and guidelines for the design of floating systems, which are consistent with the local conditions, mainly site and meteorological and oceanographic conditions, operating conditions, production volumes, economics, inspection and maintenance practices and philosophies. To this end, a risk assessment approach is selected as the appropriate framework for decisions making in regard to design criteria. As part of the risk assessment for structural systems, the optimal level of structural reliability and the corresponding design criteria for all relevant failure modes should be determined by consideration of the possible consequences of failures. The assessment of failure should include all relevant causes of structural failures caused by environmental extreme loading, degradation processes, accidental load events and operational errors.

Taking basis in a discussion of best practices and more recent developments on systems risks, the present paper 
introduces a generic framework for consequence assessment and risk analysis in FPSO systems. Thereafter, the scenarios considered for the risk based calibration of a design code safety format for the design of FPSO facilities in the Gulf of Mexico are outlined in some detail. Furthermore, it is shown how these scenarios may be represented in a generic risk assessment model greatly enhanced by the utilization of hierarchical risk modeling procedures such as Bayesian Probabilistic Nets (BPN's). BPN's have several advantages when compared to traditional risk assessment tools. Foremost, the risk assessment methodology will become more transparent because the considered events and their causal interrelations are represented graphically, which strongly facilitates communication of the model. Experts in design and operation of FPSOs with limited experiences in risk assessment techniques will be able to contribute directly to the basis of the risk assessment. Furthermore, BPN's may be developed generically such that they are valid for a given FPSO design concept (for example disconnectable or non-disconnectable turret, single or double hull, etc.). Information regarding specific design choices for any FPSO of the given concept type may then be introduced in the risk assessment through the nodes of the BPN's and the corresponding consequences and risk are immediately obtained without any further efforts. Finally, the BPN's greatly facilitate the identification of the weak spots in a given concept by allowing to identify the most probable causes of adverse events leading to consequences. This can provide insightful information on how risks may be better reduced by optimization of the design parameters.

In addition, to account for societal acceptance criteria of the risk associated with the operation of FPSO's, it is necessary to establish the optimal and acceptable level of reliability of the individual components of the FPSO's. A short outline and discussion of best practices in regard to risk acceptance criteria (RAC) is therefore provided and the more recent and promising concept of the Life Quality Index (LQI) as a practically applicable means to determine how much should be invested into life saving activities. This process of design optimization may then be efficiently facilitated by use of the developed risk models, the BPN's and the concept of the LQI. Finally, to illustrate how the developed approaches may be used in practical applications, the calculation of the target reliability index for the ultimate limit states (ULS) of FPSO mooring lines is presented.

\section{ON BEST PRACTICE RISK ASSESSMENT}

Reviews of the present practice on engineering safety management and risk informed decision making may be found in [1], [2], [3], [4] and [5]. Most regulated risk assessments are built up around the risk assessment process as defined e.g. in the Australian New Zealandic code AS/NZS 4360 [6] but also in the presently developed ISO standard on "General principles on risk assessment for structures”. Following this process, the risk analysis may be represented in a generic format, which is largely independent from the application, e.g., independent on whether the risk analysis is performed in order to document that the risks associated with a given activity are acceptable or whether the risk analysis is performed to serve as a basis for a management decision.

It is not within the scope of the present paper to outline best practice risk assessment in any detail. Instead, some of the more recent insights derived from practical applications as well as from research in the area of risk assessment are presented and discussed in the following. This discussion will then form part of the basis for the presentation of the risk assessment models utilized for the risk based development of a design code safety format for FPSO installations in the Mexican sector of the Gulf of Mexico.

\section{On the identification of systems and scenarios}

Practical experience has shown that one of the main problems leading to inconsistent risk assessments originates from incomplete system representations. Only too often it is seen that different risk assessments, even of the same activity, implicitly or explicitly are based on different basic assumptions; this concern especially the modeling of consequences, where a clear definition as to what is to be included and what not is often missing. Furthermore, with respect to the treatment of uncertainties and of dependences between the events and scenarios leading to the consequences, many risk assessments are performed on a very diverging level of detail and accuracy. The experiences made in the profession during recent years, however, indicates that risk assessments may effectively be regulated and homogenized through appropriate definition of how to identify and represent the systems being analyzed, see e.g. [7].

To counteract such problems, it has become best practice in many industrial risk assessment practices to perform risk screening meetings; workshops where a broad representation of expertise of relevance for the risk assessment is ensured. The main purpose being to gather all available information and knowledge about the considered system on a subjective and or semi-quantitative basis and to narrow down the scope of the detailed quantitative risk assessment. Risk assessment procedures and tools should thus be developed or adapted to support the consistent identification of the considered system and the relevant scenarios.

\section{On the assessment of consequences}

In connection with the assessment of consequences of loss of lives, controversy and inconsistency is observed throughout the profession, see e.g. [8]. In some risk assessments it is seen that loss of lives are accounted for in risk based cost-benefit evaluations by associating lost lives with monetary values 
representing the societal value of a life, assessed on very different bases. Recent research has, however, pointed to the perspective that this need not be done at all [9]; the issue being, that such conversions are only relevant in situations where compensations are to be paid. The compensations are generally regulated by the practice of law [1].

Another problem relating to consequence assessments concerns the modeling of especially rare events with potentially very large consequences. Traditionally such events have been accounted for in risk assessments and regulations through an increased weighing of the risks resulting from events associated with large consequences relative to events associated with low consequences. This phenomenon is typically referred to as risk aversion and, insofar that the weighing is not based on detailed quantitative assessments but rather on conservative and subjective evaluations, it may lead to great inconsistencies in the risk assessment and even to ethical inconsistencies when consequences are associated with loss of lives. To circumvent the need for and use of risk aversion in risk assessments, the concept of so-called indirect consequences [10] was developed. Such consequences aim at extending the traditional consequence analysis to specifically consider consequences going beyond the boundaries of the considered activity or facility in terms of time and space but also to account for possible consequences triggered by the public opinion in the aftermath of severe events [8].

\section{On the analysis of probability}

Some discussions have been ongoing in the last decade on the treatment of uncertainties in risk assessment. This specifically concerns what is now referred to as natural variability (aleatory uncertainty) and lack of knowledge and data (epistemic uncertainty), see e.g. [11], [12], [13] and [14].
Within the area of probabilistic seismic hazard analysis and more recently in the context of assessing the consequences of global warming it is customary to specifically account for the different types of uncertainty in order to systematically represent different expert opinions on relevant model hypothesis. It has been shown in numerous applications that the differentiation between the different types of uncertainties is crucial in the context of systems analysis, in the analysis of extreme events over time and space, in portfolio risk management, but also in the context of risk informed decision making where the option to collect additional knowledge is considered a potential risk reducing measure.

To facilitate a consistent treatment of uncertainties socalled hierarchical probabilistic models have been found appropriate; especially Bayesian hierarchical models, see e.g. [14]. In addition, compared to traditional quantitative risk assessments Bayesian risk models facilitate straightforward updating based on any new evidence and direct inclusion of subjective expert knowledge.

\section{GENERIC MODELS FOR CONSEQUENCE ASSESSMENT}

As mentioned before the system representation is a key factor in regulating risk assessments. However, best practices in risk assessment do not appreciate this systematically and in the different application areas there are very diverging schemes for identifying and defining the system. In fact, the so-called generic procedure for risk assessment [6] does not at all address the representation of the system. With this starting point, the Joint Committee on Structural Safety (JCSS) developed a document on risk assessment in engineering with a special address on the specification of a generic framework for representation of the systems subject to risk assessment, see [7].
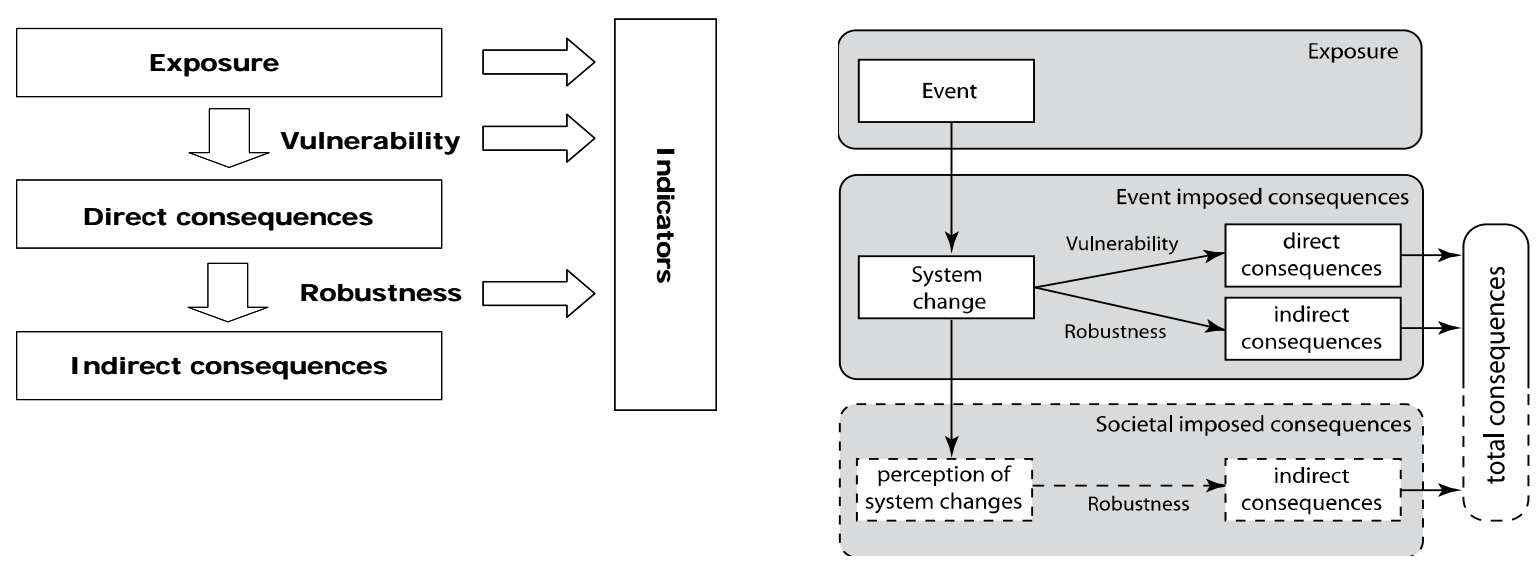

Figure 1. Illustration of the system representation suggested by the JCSS (2008) 


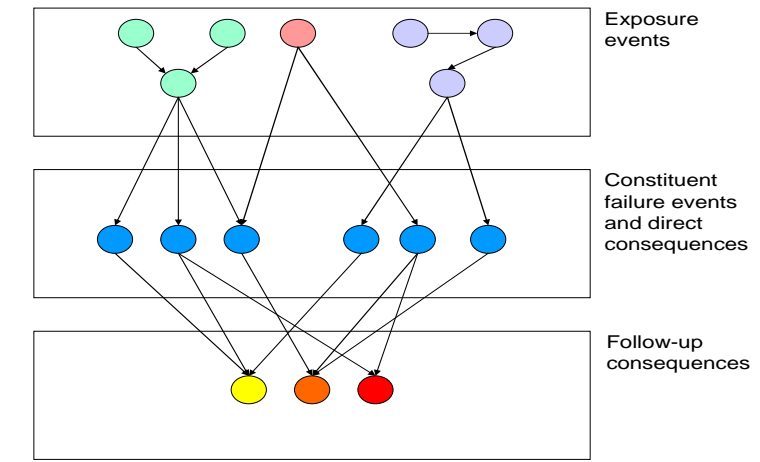

Figure 2. Logical representation of interrelation betweenexposures, constituent failures, sequences of constituent failures and consequences

In Figs.1 and 2, the basic approach behind the system representation is illustrated. The system is represented through three different characteristics; exposures, direct consequences and indirect consequences see Fig. 1. The technical and procedural components of a given system are represented by so-called constituents who in their interaction provide the functionalities of the system itself, see Fig. 2. The risk model is formulated such as to accommodate for any information available in regard to the facility being assessed through socalled indicators. The potential hazards are modeled by different exposure events acting on the constituents of the facility, see Fig. 2. The constituents of the facility can be considered as the facility's first defense in regard to the exposures. These constituents are conveniently represented by limit state functions defining the events of failure and survival of the structural element respectively. Damages to the constituents correspond to direct consequences. Direct consequences may comprise monetary losses, loss of lives, damages to the qualities of the local environment or just changed characteristics of the constituents. Based on the combination of events of constituent failures and corresponding consequences, indirect consequences may occur; typically in some form of loss of system functionality and derived effects thereof. The indirect consequences in risk assessment play a major role. Typically the indirect consequences evolve spatially beyond the boundaries of the facility and also have a certain, sometimes postponed, development in time [10].

This generic indicator-based system representation methodology has been utilized successfully in various offshore industrial applications including the risk based development of design basis considered in the present paper and repair planning for offshore platforms [15].

\section{FPSO CONSEQUENCE AND SCENARIO MODELING}

In establishing a risk based design code safety format for FPSO structures, the general risk assessment serves as a means for the calibration of safety factors for different FPSO concepts under given operating conditions, i.e., as a function of water depth and environmental conditions.

As outlined in the foregoing, an important task in the risk assessment is the identification of the considered system in regard to relevant scenarios and consequences. Taking basis in the generic indicator based system representation by the JCSS [7] adapted to the present purposes as illustrated in Fig. 3, the FPSO system and relevant scenarios were identified at risk screening meetings. In this process the common practice for risk assessments of FPSO systems was considered for as well as available statistics on accidents and failures of FPSO systems. However, as the common practice, which rests heavily on generic failure rate data does not consider risks due to structural failures in sufficient detailing, the use of engineering expert knowledge constitutes an important part of the scenario identification.

\section{CONSIDERED CONSEQUENCES AND SCENARIOS}

In principle any event is considered which may be associated with non-negligible consequences in terms of:

$$
\begin{array}{ll}
>\quad \text { Monetary losses } \\
>\quad \text { Datalities and injuries } \\
>\quad \text { Damages to the qualities of the environment }
\end{array}
$$

The different types of consequences may arise from any technical or operational failure event, whether these are caused by extreme environmental conditions, unforeseen operational conditions or human and organizational failures. Monetary losses may be induced by material damages (including loss of facility), loss of production, compensation of fatalities and injuries, compensation due to damages to the environment as well as loss of reputation. Fatalities and injuries are considered only for the facility as a whole, i.e., in that respect there is no differentiation of the different areas on the vessel. Furthermore, due to the remote location of the operation vessels, no persons that are not associated with the operation are exposed. Third party persons, i.e., persons that are not authorized by the operator, are not considered relevant for this risk assessment. Damages to the qualities of the environment are, for the sake of simplicity, only measured in terms of volumes of releases of petrochemicals. However, it is differentiated between failure events leading to high release rates and failure events leading to small release rates due to the fact that events of the latter type might be extremely difficult to detect and thus might over long time induce significant undetected damage. 


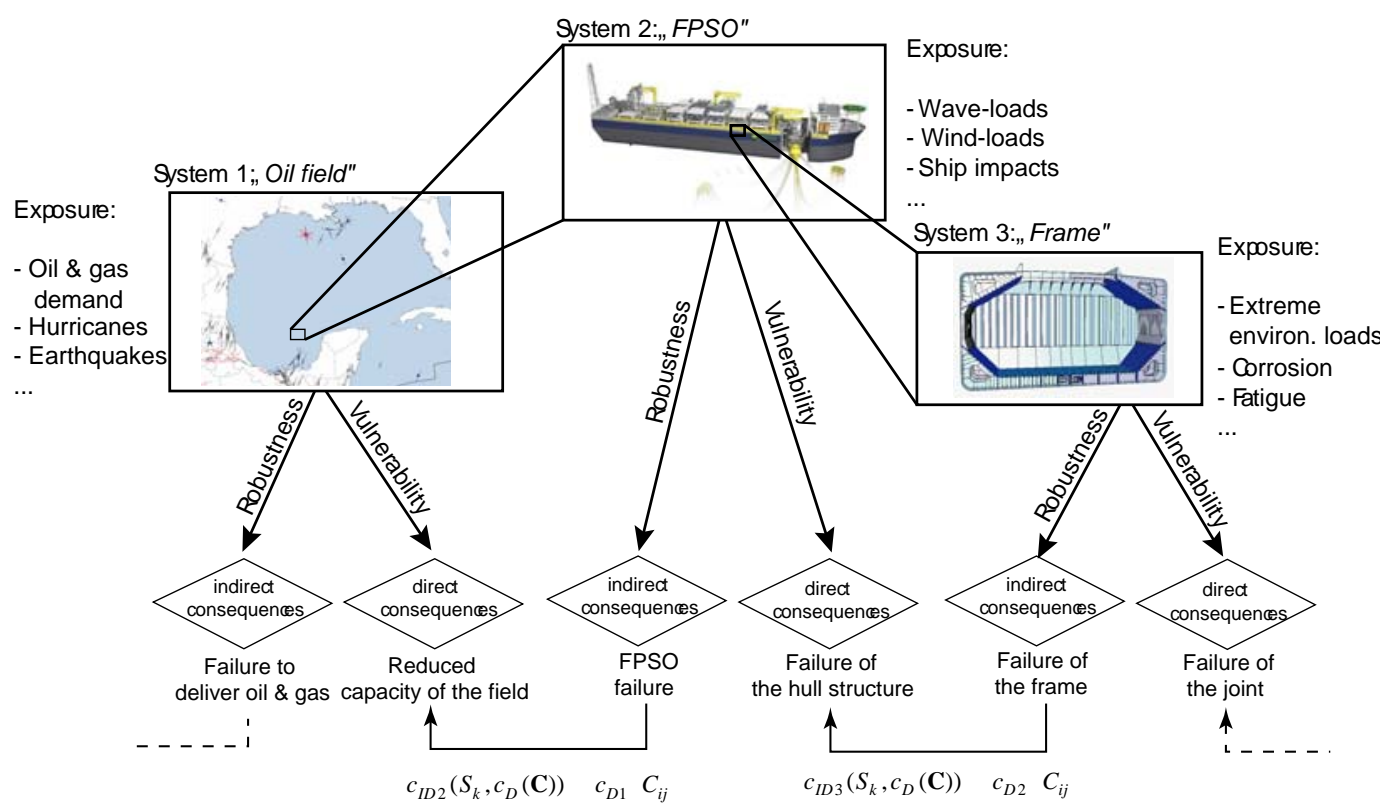

Figure 3. Generic system characterization in different scales in terms of exposures, direct and indirect consequences

While fatalities and injuries, as well as environmental damages, lead to monetary consequences, as discussed above, they must also be considered separately because acceptance criteria are typically specified for each of these categories separately.

The failure events considered have causes in the following different types of exposure events:

$>$ Environmental loads on hull, mooring system and risers

- extreme and normal environmental loads due to wind, waves and current

$>$ Operational loads in the process/risers and on the hull

- pressure and temperature

- storage and offloading of tanks

- ballasting operations

Human/operational errors

- collision with passing vessels

- helicopter crash

- dropped objects

- tank cleaning and hot works

In general the immediate effect of the exposure event will be either the development of fires and explosions or structural damages. These states are considered damage states in the risk modeling, i.e., states associated with direct consequences. The following direct consequences are considered:

$>$ Cost of repair and replacement

$>$ Fatalities and injuries (including compensation)

$>$ Release of petrochemical to the environment

Finally, as a consequence of the considered damage states, indirect consequences might occur. In the present context the following indirect consequences, which are all associated with monetary losses, are considered:

$>$ Loss or closure of production

$>$ Loss of vessel

$>$ Loss of reputation

As the present interest is in establishing a framework and tools for the assessment of risks in general as well as the impact of different FPSO concepts and design criteria on risks, the differentiation of events related to consequences illustrated in Fig. 4 is introduced. In regard to the events in the red zone of Fig. 4, i.e., events originating from the process of the vessel, the risk takes basis in generic data obtained from the literature. Thus for these risks no detailed risk assessment and consequently no specific consequence models will be developed. However, the orders of magnitude of the risks from these events as obtained from generic data allows for an assessment of the significance of these risks as compared to other risks on the vessel.

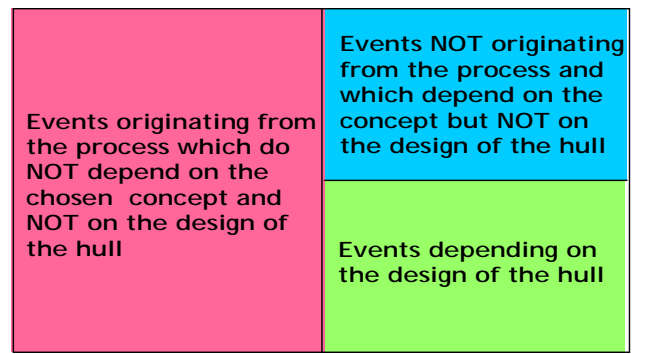

Figure 4. Differentiation of events associated with consequences 
The events in the blue zone of Fig. 4 specifically relate to events initiating from the mal-performance of the riser system and the mooring system as will be analyzed and considered in detail in Sections 6-7. Finally, the events in the green zone are events directly associated with the criteria applied for the design and maintenance of the vessel hull structure. For what concerns the consequence modeling of the event of the green and the blue zone, the illustration given in Fig. 5 facilitates the identification of events associated with consequences. In the following only the consequences and scenarios of relevance for the calibration of safety factors for the design of the mooring system, i.e. extreme load events will be provided, however, in the performed studies also normal environmental load conditions and deterioration failure modes were addressed in detail.

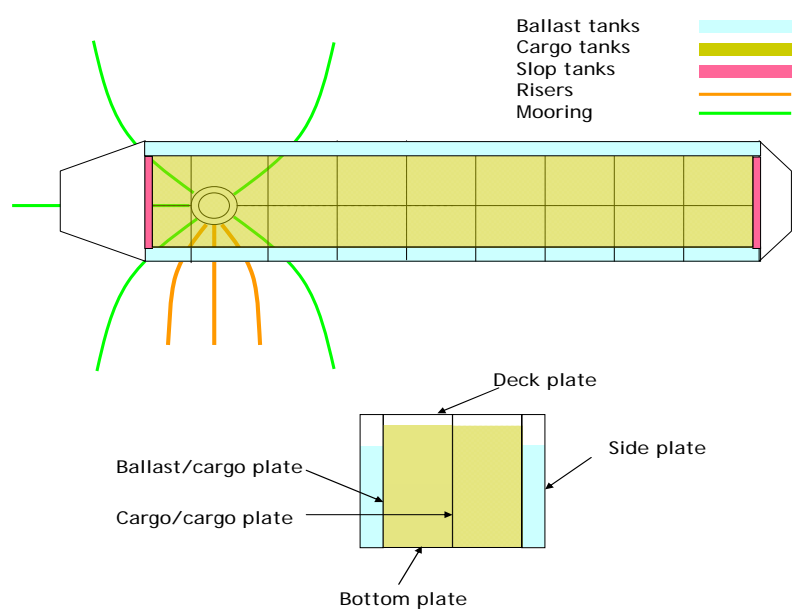

Figure 5. Illustration of the constituents of the ship hull, riser and mooring system

\section{$\begin{array}{lll}\text { SCENARIOS RELATED TO } & \\ \text { ENVIRONMENTAL LOAD CONDITIONS }\end{array}$}

EXTREME

The following description of scenarios and consequences follows the generic systems representation described in Section 3 and specifically addresses exposure events, damage states, direct and indirect consequences. In the following only the details regarding the extreme storm event of relevance for ultimate limit state (ULS) safety format calibration will be provided as this is the scenario considered in the example in Section 7.

\section{Extreme storm event scenarios}

In the development of the following scenarios, the FPSO hull, risers and moorings are generally assumed intact but subject to possible effects of degradation due to fatigue and corrosion. Furthermore, the extreme environmental loads affect the response of the FPSO system in dependence of ballast and cargo operational conditions.
The following events are considered associated with direct consequences:

1a) local load effects cause failure of scantlings and/or global load effects exceed the sectional capacity of the hull, $1 b)$ one or more mooring lines fail due to combined effect of fatigue and extreme loads,

1c) green water on deck with resulting damages on topside structures,

1d) cracks develop with resulting loss of containment between tanks or between tanks and sea,

1e) one or more risers fail due to combined effect of fatigue and extreme loads,

1f) due to evacuation and well closure the production is interrupted

The listed failure events lead to direct consequences in terms of monetary losses, damages to the qualities of the environment, fatalities and injuries. The consequences to personnel depend on the availability and function of gas detection equipment, fire fighting system, evacuation and life saving systems.

The following events are associated with indirect consequences:

2a) due to local or global overloading of the capacity of the ship hull the vessel founders and may be lost

2b) due to loss of mooring system the vessel drifts, mooring chains may damage sub-sea systems and the vessel may founders and be lost

2c) due to crack growth in tank partitions explosions in the cargo area may occur which in turn may lead to loss of vessel

2d) due to damages and/or overloading of the topside structures modules might fall on deck, initiate explosions and may lead to loss of vessel

2e) due to large damages/loss of vessel and/or significant damage to the environment, the production on other vessels might be seized for a period of time while investigations are undertaken

2f) after the storm, due to damages and associated repair works, the production will be delayed

$2 \mathrm{~g}$ ) major irregularities or environmental damages lead to loss of reputation

The listed indirect consequences include monetary losses, damages to the qualities of the environment, fatalities and injuries. The consequences to personnel depend on the availability and function of gas detection equipment, fire fighting system, evacuation and life saving systems.

\section{RAC - THE LIFE QUALITY INDEX}

Over the last 10-15 years significant developments have been made based on which regulation of risk criteria may now be supported by, if not directly based on, scientific socio- 
economical models. This is discussed in [16], [17] and later in [18].

As a variant of the so-called marginal life saving cost principle, see e.g. [17], the Life Quality Index (LQI) is proposed in [16]. The LQI takes basis in a modelling of societal preferences for investments into life safety in dependency of macro-economical indicators. Based on this preference it is possible to determine whether an activity or facility is acceptable or not, taking into account the efforts made to reduce life safety risks.

The LQI models the preferences of a society quantitatively as a scalar valued social indicator comprised of a relationship between the part of the GDP per capita which is available for risk reduction purposes $g$, the expected life at birth $l$ and the proportion of life spend for earning a living $w$.

The Life Quality Index can be expressed in the following principal form:

$$
\operatorname{LQI}(g, l)=g^{q} l
$$

The parameter $q$ is a measure of the trade-off between the resources available for consumption and the value of the time of healthy life. It depends on the fraction of life allocated for economical activity and furthermore accounts for the fact that a part of the GDP is realized through work and the other part through returns of investments. The constant $q$ is assessed as:

$$
q=\frac{1}{\lambda} \frac{w}{1-w}
$$

where $\lambda$ is a constant taking into account that only part of the $G D P$ is based on human labor, the other part is due to investments. Every risk reduction measure will affect the value of the LQI. The consideration that any investment into life risk reduction should lead to an increase of the LQI leads to the following RAC [7]:

$$
\mathrm{d} C_{y}(p) \geq-\frac{g}{q} C_{x} N_{P E} k \mathrm{~d} m(p)
$$

where $p$ is the design variable, i.e., the possible decision alternatives for risk reduction, $C_{y}(p)$ are the annual investments which should be invested into life safety, $m(p)$ is the failure rate, $C_{x}$ is a demographical economical constant corresponding to a given scheme $x$ for mortality reduction, $N_{P E}$ is the number of persons exposed to the failure, and $k$ is the probability of dying given a failure. Eq.3 states that risk reduction measures must be undertaken as long as the corresponding marginal risk reduction exceeds the marginal costs of risk reduction.

In Fig. 6 it is illustrated how the failure rate $m(p)$ as well as the normalized risk reduction costs $C_{y}(p) q / C_{x} N_{P E} k g$ depend on $p$. In the illustration it is assumed that the cost of risk reduction is linear in the decision parameter $p$. The failure rate $m(p)$, on the other hand, is in general a non-linear function of $p$. The maximum acceptable failure rate is obtained for the minimum value of $p$ for which the above inequality holds, assuming that the failure rate decrease continuously with increasing $p$. Any value of $p$ larger than this value can be considered acceptable.

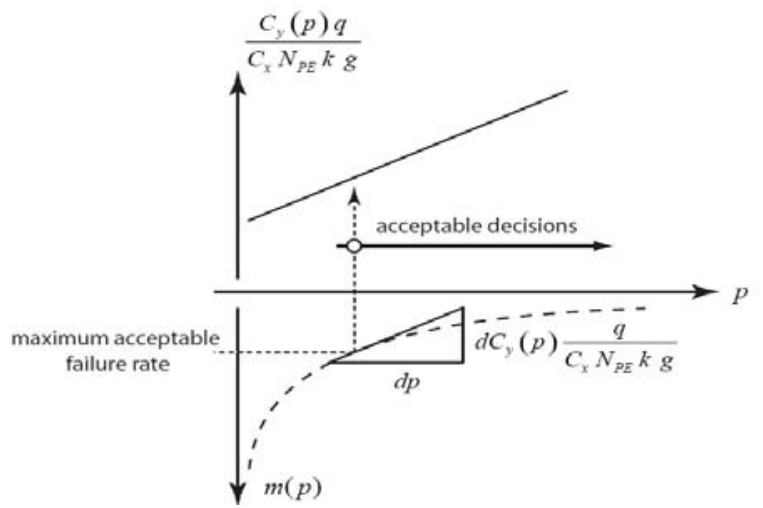

Figure 6. Illustration of the use of the risk acceptance criterion (RAC)

\section{BAYESIAN NETWORK FOR MOORING LINE ULTIMATE LIMIT STATE}

BPN's constitute a flexible, intuitive and strong model framework for Bayesian probabilistic analysis [19]. BPN's may replace both fault and event trees and can be used at any stage of a probabilistic analysis. Due to their mind mapping characteristic, they comprise a significant support in the early phases of a probabilistic analysis, where the main task is to identify the potential scenarios and the interrelation of events leading to adverse events. Applications of BPN's for offshore applications are described in e.g. [20] and [15].

Following [21], the general principle of the BPN employed for the risk assessments in the present study is outlined in Fig. 7. The constituent failure events are described by the limit state functions, which include the selected safety factors as variables. The financial consequences are then computed as the sum of the direct and the indirect consequences. These include various types of economical consequences, such as lost production, repair/replacement cost, compensation payments, loss of reputation etc. These consequences are the economical costs arising to the operator, on the basis of which the optimization of the target reliability (and corresponding safety factors) is performed. Because the 
BPN assesses the expected utilities, all consequence nodes must be in the same unit. This unit is typically a monetary value. For this reason, the consequences to personnel, which are fatalities and injuries, and the consequences to the environment, here oil spills, must be translated to monetary values.

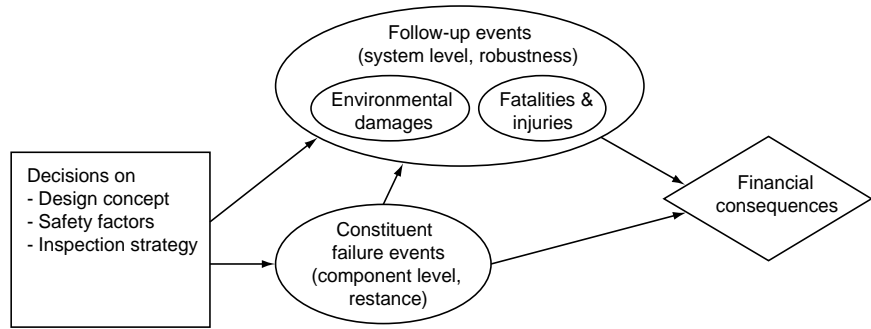

Figure 7. General format of the Bayesian networks applied for the risk assessment relating to the mooring line design

The nodes in the BPN describing adverse events to personnel and to the environment, which are part of the indirect consequences, provide the necessary information to check acceptability of the safety factors, in accordance with the overall risk acceptance criteria. To determine the acceptance criteria associated with injuries and fatalities, the approach based on the Life Quality Index is chosen, as outlined in Section 5.

The probability of occurrence of the structural failure (including deterioration failures) is obtained directly from the structural reliability computations performed using a response surface approach, as a function of the design safety factors [22].

To assess the risk related to a single constituent failure event, it is required to compute the expected consequences, involving both direct and indirect consequences. To assess the direct consequences, it is in most cases sufficient to consider a constituent failure event individually. To determine the expected indirect consequences, however, it is generally necessary to assess the expected consequences for a combination of failure events, in particular when different failure events exhibit strong stochastic or functional dependence.

The proposed BPN for the case of mooring line failures due extreme environmental events is shown in Fig. 8. The BPN gives the expected failure costs and takes into account likelihood of loosing one or more than one mooring lines and the influence of this failure on the mooring system and on the whole FPSO.

The decision variable in the BPN is the mooring line annual reliability index $\beta$ (or alternatively the corresponding probability of failure). Given the failure of a single (or several) mooring line, there is an increased probability of failure of the mooring system as a whole. In turn, the state of the FPSO (operational, damage at location, damaged and moved, lost) depends on the state of the mooring system. Finally, depending on the damage state of the FPSO there are different consequences: loss of lives, oil spills and damage to other installations. These damage states are associated to the following costs: cleaning, compensation, loss of reputation, production loss and repairing. The costs are included in the BPN model, based partly on the information provided in [23] and on the experience of designers and operators.

The BPN shown in Fig. 8 allows to explicitly to model all dependences in the process. As an example, the amount of oil spill depends not only on the state of the FPSO, but also on the success of the planned shutdown of production. Likewise, the loss of lives is dependent also on the success of evacuation of personnel during an extreme event. As stated earlier, the possibility of the floating vessel damaging other installations in the field is considered. In particular the mooring lines that are dragged along a vessel can damage installations on the sea-bed. Furthermore, there is a possibility of the vessel colliding with other installations. It is noted that in this BPN it is assumed that no deterioration (or only negligible deterioration) takes place in the system.

A strategy for reducing the probability of system failure conditional on the failure of one (or more) of the mooring lines is to supply additional strength. However, considering the potentially strong correlation among failure events, it should be clear that adding additional strength for the ULS without any additional measures is an inefficient strategy against structural damage.

\section{CASE STUDY - MOORING LINE RELIABILITY}

In the following the example from [21] is outlined for the purpose of illustrating how the developed risk assessment framework and the specific models for risk analysis of FPSO structures can be utilized for purposes of code safety format calibration. In this respect the particular issue of assessing the target reliability for the components of the mooring system is considered.

The BPN shown in Fig. 8 is used to estimate expected consequences due to failures of mooring lines. Correspondingly, as an example, Fig. 9 presents the BPN together with the probabilities of all states of all nodes given failure of a single mooring line due to overloading. Here, the total expected cost is estimated as 29 Million US\$. The probability of mooring system failure in this case is very high (70\%), because for the case of ULS the performances of the individual mooring lines are highly correlated, due to the common loading and not least because of the cascading effect of failures. This corresponds to observations made elsewhere [23]. The main component to the expected cost of failure is the 
cost of production loss/repair (27 Million US\$). The expected cost due to damages to other installations is 1.5 Million US\$.
The contributions of other cost sources are relatively low, as can be seen in Fig. 9.

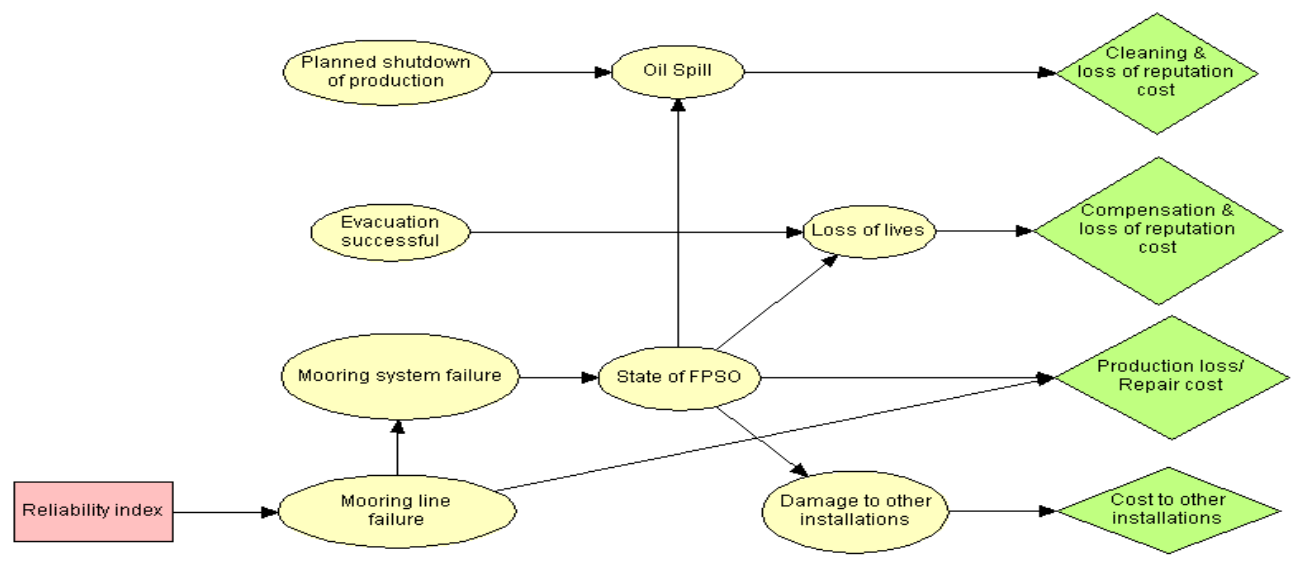

Figure 8. The Bayesian Probabilistic Network applied for the optimization and assessment of acceptability of mooring line reliability for ULS design

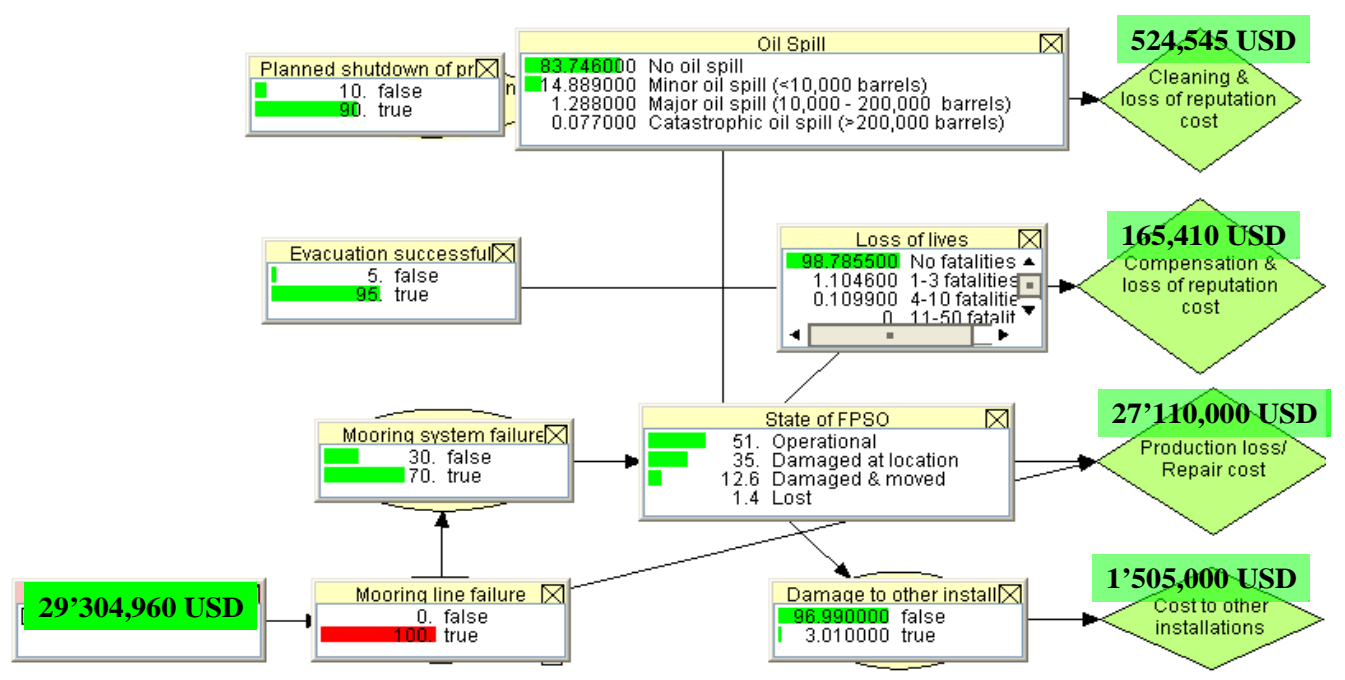

Figure 9. The BPN for mooring line risk analysis providing the marginal probabilities of system state variables and the expected value of the consequences given failure of one mooring line due to overloading

Based on information obtained from manufacturers, the initial costs $C_{I}$ of the mooring system (design, materials, transportation, installation, etc.) are modeled as a function of the annual mooring line probability of failure:

$$
C_{I}=20-2.2 \ln \left(P_{F}\right) \text { [millions of US\$] }
$$

Total expected costs are obtained summing expected failure costs (in present value) $E\left[C_{F}\right]$ and the mooring line system initial costs:

$$
E[C]=C_{I}+E\left[C_{F}\right]
$$

In order to calculate the expected consequences in present value, we use the following expression (assuming that the annual mooring system reliability is constant):

$$
E\left[C_{F}\right] \approx C_{F E} P_{F} V
$$

where $C_{F E}$ are the expected consequences of a mooring line failure event (results from the BPN, given the failure of mooring lines), $P_{F}$ is the annual mooring system probability of failure, and $V$ is a present value factor, defined as 


$$
V=\frac{1-\exp (-r L)}{r}
$$

For this application, service lifetime is taken as $L=25$ years, and the net discount rate is set equal to $r=0.05$.

Using results from the BPN and using Eqs. 4 to 7, the total expected cost can be estimated. Results are shown in Fig. 10 . For the considered case, the optimum mooring system annual design reliability index is $\beta=2.51\left(P_{F}=6 \times 10^{-3}\right)$.

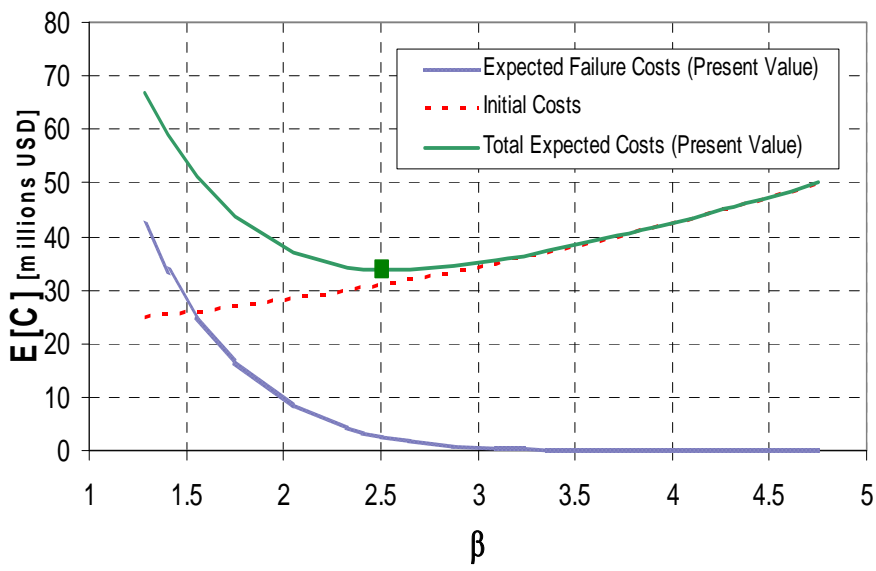

Figure 10. Optimal annual reliability index for mooring system components

The minimum acceptable mooring line system reliability index is calculated by means of the LQI criterion, Eq. 3. The following parameters were used: $N_{P E}=50, k=1$ (this value is highly conservative), $g=0.4 \mathrm{GDP}=3200 \quad$ US $\$$ (GDP=8000US $\$$ ), $\lambda=0.8, w=0.2, C_{x}=19$. Annual investment in life safety, $C_{y}(p)$, is obtained by dividing $C_{I}$ (Eq.4) by $L=25$ years. Substituting this data in Eq.3 an annual target probability equal to $P_{F}=8.85 \times 10^{-3}$ and a corresponding reliability index $\beta=2.37$, are obtained. Thus, the optimal index $\beta=2.51\left(P_{F}=6 \times 10^{-3}\right)$ is acceptable and can be taken as the design target reliability.

It is important to note that the minimum acceptable reliability index based on the LQI is strongly dependent on economic and societal parameters, which may change significantly from country to country. Consider, for instance, that the FPSO is to be located in Norwegian waters. In that case $g=14149$ US\$ and $w=0.1$ [18]; then, keeping the other parameters unchanged, $P_{F}=8.97 \times 10^{-4} \quad(\beta=3.12)$. Hence $P_{F}=6 \times 10^{-3} \quad(\beta=2.51)$ obtained from minimizing expected costs is not an acceptable probability of failure, and $P_{F}=8.97 \times 10^{-4}$ would be the design target. Note that this target is not necessarily equal to the usual target value of $10^{-4}$ because of two main reasons: (1) the minimum reliability index based on LQI depends not only on economic and societal issues, but also on the failure rate (structure specific), on the number of people exposed, and on the probability of life loss given a failure; and (2) the target reliability index for design is defined taking into consideration both the RAC criterion and the optimum risk-based reliability index, which is structure specific (obtained by means of BPN in this work).

\section{CONCLUSIONS}

Based on a recent industrial project a risk-based methodology is presented for the purpose of risk based design safety format calibration for FPSO systems in the Mexican sector of the Gulf of Mexico. Special emphasis is given to improve the traditional approaches to quantitative risk assessments by utilization of recent advances in representation of systems, scenario, consequence modeling and definition of risk acceptance criteria. To this end the indicator based generic system representation recently developed by the Joint Committee on Structural Safety (JCSS) is utilized and adapted to identified relevant failure scenarios. Furthermore, on the basis of developed risk models implemented and analyses using Bayesian Probabilistic Nets together with the the Life Quality Index (LQI) principle, acceptable reliability indexes for mooring line design for FPSO's systems are identified. The optimum design reliability index is obtained by minimization of the total expected costs, whereby a Bayesian Probabilistic Net (BPN) is used to compute the expected failure cost. In order to guarantee that design reliability index is acceptable from a societal point of view, a minimum acceptable reliability index is calculated by means of a criterion which takes into account the estimated life risks as well as the efficiency of life saving activities in the assessment of acceptability.

As an illustration of the application of the methodology, the determination of the target reliability index for the ultimate limit state of tension failure under extreme environmental events is considered. For the investigated case, the optimum reliability index (annual reliability) is computed as 2.5 with a minimum acceptable value of 2.4. Although these values are site and structure specific, they are significantly lower than the values commonly used in practice. They are also lower than the values suggested by the JCSS model code [24]. However, it is noted that these values depend on the model for the design cost of the mooring line, which is only a rough estimate. This model should be verified before any definite conclusions can be made.

\section{REFERENCES}

[1] Faber M.H., Stewart M.G. (2001). Risk assessment for civil engineering facilities: critical overview and discussion. Reliability Engineering \& System Safety, 80 (2), May, pp. 173-184. 
[2] Ale B.J.M. (2002). Risk assessment practices in The Netherlands. Safety Science, 40, pp. 105-126.

[3] Ale B.J.M. (2005). Tolerable or acceptable: a comparison of risk regulation in the United Kingdom and in the Netherlands. Risk Anal, 25 (2).

[4] Trbojevic V. (2005). Risk criteria in EU. Proc. to European Safety and Reliability Conference, Tri City, Poland.

[5] Hartford D.N.D. (2008). Legal framework considerations in the development of risk acceptance criteria. Structural Safety, in press.

[6] AS/NZS 4360 (1999). Risk Management. Standards Australia, Sydney.

[7] JCSS (2008). Risk Assessment in Engineering, Principles, System Representation \& Risk Criteria, ISBN 978-3909386-78-9.

[8] Schubert M., Faber M.H., Baker J. W. (2007). Decision making subject to aversion of low frequency high consequence events. Special Workshop on Risk Acceptance and Risk Communication, Stanford, USA, March 26-27.

[9] Rackwitz R., Lentz A., Faber M.H. (2005). Socioeconomically sustainable civil engineering infrastructures by optimization. Structural Safety, 27 (3), July, pp. 187-229.

[10] Faber M.H., Maes M.A. (2004). Modeling of risk perception in engineering decision analysis. Proc. 11th IFIP WG7.5 Working Conference on Reliability and Optimization of Structural Systems, pp. 95-104.

[11] Faber M.H. (2005). On the treatment of uncertainties and probabilities in engineering decision analysis. Journal of Offshore Mechanics and Arctic Engineering, Trans. ASME, 127(3), August, pp. 243-248.

[12] Faber M.H., Maes M.A. (2005). Epistemic uncertainties and system choice in decision making. Proc. ICOSSAR'05, 9th International Conference on Structural Safety and Reliability, Rome, June 19-23.

[13] Der Kiureghian A., Ditlevsen O. (2009). Aleatory or epistemic? Does it matter? Structural Safety, 31, pp.105112.

[14] Nishijima K., Maes M.A., Goyet J., Faber M.H. (2009). Constrained optimization of component reliability of complex systems. Structural Safety, 31(2), March, pp. 168178.

[15] Montes-Iturrizaga R., Heredia-Zavoni E., VargasRodríguez F., Faber M.H., Straub D., De la O J. (2009). Risk based structural integrity management of marine platforms using bayesian probabilistic nets. Journal of Offshore Mechanics and Arctic Engineering, ASME, 131 (1), February, pp. 011602-1 to 011602-10.

[16] Nathwani J.S., Lind N.C. and Pandey M.D. (1997). Affordable safety by choice: The Life Quality Method. Institute for Risk Research, University of Waterloo, Ontario, Canada.

[17] Ramsberg J., Sjöberg L. (1997), The cost-effectiveness of lifesaving interventions in Sweden, Risk Analysis, 17 (4).

[18] Rackwitz R. (2005). The philosophy behind the Life Quality Index and empirical verification. Updated memorandum to JCSS.

[19] Jensen F. (2001). Bayesian Networks and Decision Graphs. Springer.
[20] Faber M.H., Kroon I.B, Kragh E., Bayly D., Decosemaeker P. (2001). Risk Assessment of Decommissioning Options Using Bayesian Networks. Proc. OMAE2001, 20th Conference on Offshore Mechanics and Arctic Engineering, Rio de Janeiro, Brazil, June 3-8, [OMAE2001/S\&R-2115].

[21] Montes-Iturrizaga R., Heredia-Zavoni E., Straub D., Faber M.H. (2008a). Optimum and minimum acceptable reliability indexes for mooring line design in FPSO Systems. Proc. IFIP WG 7.5 Working conference, Mexico City.

[22] Montes-Iturrizaga R., Silva-González F.L., HerediaZavoni E., Inda G., Straub D. (2008b). Calibration of safety factors for mooring lines. Proc. IFIP WG 7.5 Working conference, Mexico City.

[23] Health and Safety Executive (HSE) (2006). Floating production system - JIP FPS mooring integrity. Research Report 444, Health and Safety Executive, UK.

[24] JCSS (2006). JCSS Probabilistic Model Code. Joint Committee on Structural Safety (http://www.jcss.ethz.ch) 2006. 